\title{
EXPERIMENTAÇÃO EM QUÍMICA DE COMPOSTOS ORGANOMETÁLICOS: PREPARAÇÃO DO CLORETO DE DICARBONILCICLOPENTADIENILFERRO(II)
}

\author{
Zaine Teixeira, Suzan P. Vasconcellos, Luzia Koike e Gilson H. M. Dias* \\ Instituto de Química, Universidade Estadual de Campinas, CP 6154, 13084-971 Campinas - SP, Brasil
}

Recebido em 3/1/06; aceito em 16/5/06; publicado na web em 28/11/06

\begin{abstract}
PRACTICAL IN ORGANOMETALLIC CHEMISTRY: PREPARATION OF DICARBONYLCYCLOPENTADIENYLIRON(II) CHLORIDE. In this work, we describe a new method for obtaining $\left[\mathrm{Fe}(\mathrm{CO})_{2}\left[\left(\eta^{5}-\mathrm{C}_{5} \mathrm{H}_{5}\right) \mathrm{Cl}\right]\right.$ employing simple techniques and lowcost reagents. It is worth mentioning that this method is faster than others reported in the literature. It was applied in laboratory classes for undergraduate students, exploring different concepts in organometallic chemistry and discussing the steps involved in the synthetic route.
\end{abstract}

Keywords: dicarbonylcyclopentadienyliron(II) chloride; organometallic synthesis; undergraduate laboratory.

\section{INTRODUÇÃO}

Os compostos organometálicos são comumente definidos como compostos com pelo menos uma ligação metal-carbono. Estes compostos apresentam uma vasta aplicação em catálise, além de se apresentarem fascinantes em termos conceituais de estrutura, ligação e reatividade. Especificamente, o complexo $\left[\mathrm{Fe}(\mathrm{CO})_{2}\left[\left(\eta^{5}-\mathrm{C}_{5} \mathrm{H}_{5}\right) \mathrm{Cl}\right]\right.$ é um precursor conhecido de complexos ferro-alquilas $\left[\mathrm{Fe}\left(\mathrm{PPh}_{3}\right)(\mathrm{CO})\left[\left(\eta^{5}-\mathrm{C}_{5} \mathrm{H}_{5}\right) \mathrm{R}\right]\right.$ e ferro-ligantes neutros $\left[\mathrm{Fe}(\mathrm{CO})_{2}\left[\left(\eta^{5}-\right.\right.\right.$ $\left.\left.\mathrm{C}_{5} \mathrm{H}_{5}\right) \mathrm{L}\right]^{+}$. Os primeiros são amplamente usados em síntese orgânica assimétrica $^{1}$ e os $\left[\mathrm{Fe}(\mathrm{CO})_{2}\left[\left(\eta^{5}-\mathrm{C}_{5} \mathrm{H}_{5}\right) \mathrm{L}\right]^{+}\right.$são conhecidos por ativar ligantes alquenos e alquinos nas adições nucleofílicas de carbono e heteroátomos pelas reações de Michael promovidas por metais ${ }^{2}$, como catalisadores nas reações de condensação ${ }^{3}$, na síntese de carboxialquilas de ferro ${ }^{4}$ e em reações de migração induzida de ânions ${ }^{5}$. $\mathrm{O}$ método clássico ${ }^{6}$ de obtenção do $\left[\mathrm{Fe}(\mathrm{CO})_{2}\left[\left(\eta^{5}-\mathrm{C}_{5} \mathrm{H}_{5}\right) \mathrm{Cl}\right]\right.$ consiste na reação de $\mathrm{O}_{2}$ e $\mathrm{HCl}$ concentrado com $\left[\mathrm{Fe}(\mathrm{CO})_{2}\left[\left(\eta^{5}-\mathrm{C}_{5} \mathrm{H}_{5}\right)\right]_{2}\right.$. Nesta reação, no entanto, o procedimento para a remoção do ácido é lento e às vezes o produto sofre hidrólise parcial no processo final. Como consequiência, foram publicadas rotas sintéticas alternativas, usandose principalmente metais de transição oxidantes, tais como os sais de $\mathrm{Ag}^{+7}$ ou $\mathrm{Fe}^{3+8}$, ou por oxigênio molecular e ácido fluorobórico em acetona ${ }^{9}$. Evidências espectroscópicas mostram que os intermediários ou possíveis subprodutos nestas reações são os complexos $\left[\mathrm{Fe}(\mathrm{CO})_{2}\left[\left(\eta^{5}-\mathrm{C}_{5} \mathrm{H}_{5}\right)\left(\mathrm{OH}_{2}\right)\right]^{+9}\right.$ e $\left[\mathrm{Fe}(\mathrm{CO})_{2}\left(\eta^{5}-\mathrm{C}_{5} \mathrm{H}_{5}\right)\left[\mathrm{OC}\left(\mathrm{CH}_{3}\right)_{2}\right)\right]^{8}$. Neste trabalho, uma nova rota de síntese do complexo $\left[\mathrm{Fe}(\mathrm{CO})_{2}\left[\left(\eta^{5}-\mathrm{C}_{5} \mathrm{H}_{5}\right) \mathrm{Cl}\right]\right.$ foi desenvolvida pela reação de $\left[\mathrm{Fe}(\mathrm{CO})_{2}\left[\left(\eta^{5}-\mathrm{C}_{5} \mathrm{H}_{5}\right)\right]_{2}\right.$ com $\mathrm{CuCl}_{2}$. Este método ofereceu vantagens consideráveis em comparação aos procedimentos sintéticos descritos na literatura, devido a um menor tempo gasto e maior simplicidade, além do baixo custo dos reagentes. Esta rota experimental foi aplicada pela primeira vez na disciplina de Laboratório de Síntese Orgânica e Inorgânica para os alunos do $6^{\circ}$ semestre do curso de Química - Modalidade Tecnológica, ingressantes em 2002, Instituto de Química da UNICAMP.

\section{PARTE EXPERIMENTAL}

O composto dímero precursor $\left[\mathrm{Fe}(\mathrm{CO})_{2}\left(\eta^{5}-\mathrm{C}_{5} \mathrm{H}_{5}\right)\right]_{2}$ foi obtido pela adaptação do método sintético descrito na literatura ${ }^{10}$. Foram adicio-

*e-mail: dias@iqm.unicamp.br nados, com auxílio de uma seringa, $1,0 \mathrm{~mL}$ de pentacarbonilferro (7,6 mmol) e $6,4 \mathrm{~mL}$ de diciclopentadieno $(47,7 \mathrm{mmol})$ em um balão de fundo redondo de $25 \mathrm{~mL}$ contendo pedras de porcelana, acoplado a um condensador de refluxo com um tubo contendo $\mathrm{CaCl}_{2}$ comercial. Tal adição foi realizada com o acompanhamento dos monitores e docentes da disciplina, tendo em vista a elevada toxicidade destes reagentes, notadamente o pentacarbonilferro. A mistura reacional foi mantida sob refluxo por aproximadamente 2 $\mathrm{h}$, em ambiente ventilado, de preferência na capela, até que todo o pentacarbonilferro (amarelo) fosse consumido. No início da reação, manteve-se o refluxo suave para possibilitar o craqueamento do diciclopentadieno, evitando assim a decomposição do pentacarbonilferro. Após esta etapa, manteve-se a solução reacional por 30 min no balão sem a manta de aquecimento para cristalização do produto, o qual foi filtrado em funil de Büchner e lavado com pequenas porções de hexano. Foram obtidos $0,55 \mathrm{~g}$ de um sólido marrom avermelhado do $\left[\mathrm{Fe}(\mathrm{CO})_{2}\left(\eta^{5}-\mathrm{C}_{5} \mathrm{H}_{5}\right)\right]_{2} \quad(41 \%$ de rendimento).

$\mathrm{Na}$ síntese do $\left[\mathrm{Fe}(\mathrm{CO})_{2}\left[\left(\eta^{5}-\mathrm{C}_{5} \mathrm{H}_{5}\right) \mathrm{Cl}\right]\right.$, foram adicionados sob agitação magnética, em pequenas porções, $1,4 \mathrm{~g} \mathrm{de} \mathrm{CuCl}_{2} \cdot 2 \mathrm{H}_{2} \mathrm{O}$ $(8,4 \mathrm{mmol})$ a uma solução de $0,5 \mathrm{~g}$ do $\left[\mathrm{Fe}(\mathrm{CO})_{2}\left[\left(\eta^{5}-\mathrm{C}_{5} \mathrm{H}_{5}\right)\right]_{2}(1,4\right.$ mmol) em $10 \mathrm{~mL}$ de acetona. A suspensão foi filtrada em funil analítico com papel de filtro para eliminar o $\mathrm{CuCl}$. A seguir, o solvente foi removido em rotaevaporador. O resíduo foi parcialmente solubilizado em $30 \mathrm{~mL}$ de clorofórmio e filtrado em coluna de vidro preenchida com alumina ( $2 \mathrm{~cm}$ de diâmetro, $2 \mathrm{~cm}$ de altura). Finalmente, todo material volátil foi removido no rotaevaporador e o produto lavado com hexano.

Foram obtidos $0,36 \mathrm{~g}$ de um sólido vermelho, o $\left[\mathrm{Fe}(\mathrm{CO})_{2}\left(\eta^{5}-\right.\right.$ $\left.\mathrm{C}_{5} \mathrm{H}_{5}\right) \mathrm{Cl}$ ], com $61 \%$ de rendimento reacional, correspondendo ao valor médio obtido pelos alunos da disciplina. Os produtos foram caracterizados quanto a seus pontos de fusão e espectros vibracionais na região do infravermelho.

\section{RESULTADOS E DISCUSSÃO}

O mecanismo de reação do $\left[\mathrm{Fe}(\mathrm{CO})_{2}\left(\eta^{5}-\mathrm{C}_{5} \mathrm{H}_{5}\right)\right]_{2}$ é descrito em referência da literatura ${ }^{11}$. Para a obtenção do complexo $\left[\mathrm{Fe}(\mathrm{CO})_{2}\left(\eta^{5}-\right.\right.$ $\left.\mathrm{C}_{5} \mathrm{H}_{5}\right) \mathrm{Cl}$ ] empregando $\mathrm{CuCl}_{2}$, propõe-se que a reação envolva um processo oxidativo com a fragmentação do $\left[\mathrm{Fe}(\mathrm{CO})_{2}\left(\eta^{5}-\mathrm{C}_{5} \mathrm{H}_{5}\right)\right]_{2}$, seguindo-se o ataque nucleofílico do $\mathrm{Cl}^{-}$(Figura 1) 


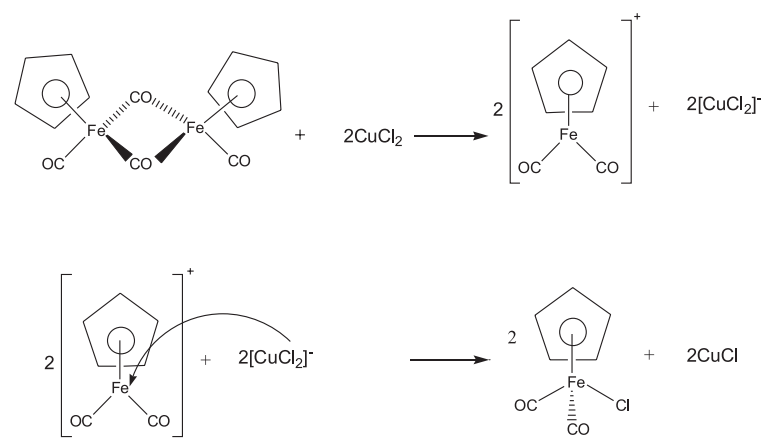

Figura 1. Mecanismo de reação para a formação do $\left[\mathrm{Fe}(\mathrm{CO})_{2}\left(\eta^{5}-\mathrm{C}_{5} \mathrm{H}_{5}\right) \mathrm{Cl}\right]$

A Figura 2 apresenta os espectros vibracionais na região do infravermelho dos complexos $\left[\mathrm{Fe}(\mathrm{CO})_{2}\left(\eta^{5}-\mathrm{C}_{5} \mathrm{H}_{5}\right)\right]_{2}$ e $\left[\mathrm{Fe}(\mathrm{CO})_{2}\left(\eta^{5}-\right.\right.$ $\left.\mathrm{C}_{5} \mathrm{H}_{5}\right) \mathrm{Cl}$. As atribuições tentativas ${ }^{12}$ (Tabela1) mostram que o $\left[\mathrm{Fe}(\mathrm{CO})_{2}\left(\eta^{5}-\mathrm{C}_{5} \mathrm{H}_{5}\right) \mathrm{Cl}\right]$ pôde ser obtido com elevada pureza, tendo em vista a ausência da banda de estiramento $\mathrm{CO}$, ligado em ponte, em $1757 \mathrm{~cm}^{-1}$.

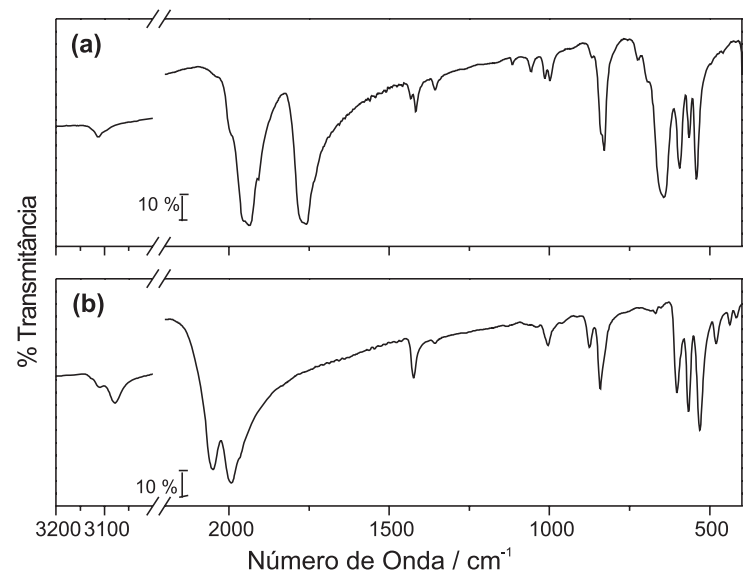

Figura 2. Espectros IV dos compostos: (a) $\left[\mathrm{Fe}(\mathrm{CO})_{2}\left(\eta^{5}-\mathrm{C}_{5} \mathrm{H}_{5}\right)\right]_{2} ;($ b) $\left[\mathrm{Fe}(\mathrm{CO})_{2}\left(\eta^{5}-\mathrm{C}_{5} \mathrm{H}_{5}\right) \mathrm{Cl}\right]$

A espectroscopia de RMN de ${ }^{13} \mathrm{C}$ e ${ }^{1} \mathrm{H}$ também é comumente empregada na caracterização destes compostos organometálicos, observando-se os deslocamentos químicos: ${ }^{13} \mathrm{C} \mathrm{CO}=213,3 \mathrm{ppm}$, $\mathrm{C}_{5} \mathrm{H}_{5}=85,9 \mathrm{ppm} \mathrm{e}{ }^{1} \mathrm{H}-\mathrm{C}_{5} \mathrm{H}_{5}=4,98 \mathrm{ppm}$ em relação ao $\mathrm{CDCl}_{3}{ }^{13,14}$.

Tabela 1. Atribuições nos espectros de IV dos complexos sintetizados

\begin{tabular}{lcc}
\hline & $\begin{array}{c}\text { Número de onda/ } \mathrm{cm}^{-1} \\
{\left[\mathrm{Fe}(\mathrm{CO})_{2}\left(\eta^{5}-\mathrm{C}_{5} \mathrm{H}_{5}\right)\right]_{2}\left[\mathrm{Fe}(\mathrm{CO})_{2}\left(\eta^{5}-\mathrm{C}_{5} \mathrm{H}_{5}\right) \mathrm{Cl}\right]}\end{array}$ & $\begin{array}{c}\text { Modo } \\
\text { Vibracional }\end{array}$ \\
\hline $3113(\mathrm{f})$ & $3078(\mathrm{f})$ & $\begin{array}{c}v p^{2}(\mathrm{CH}) \\
-\end{array}$ \\
\hline $1935(\mathrm{~F})$ & $1950(\mathrm{~F})$ & $v(\mathrm{CO})$ terminal \\
$1757(\mathrm{~F})$ & - & $v(\mathrm{CO})$ terminal \\
$1417(\mathrm{f})$ & $1423(\mathrm{mF})$ & $\delta(\mathrm{CO})$ ponte \\
$999(\mathrm{f})$ & $1004(\mathrm{f})$ & $\delta(\mathrm{CC})$ anel \\
$839(\mathrm{f})$ & $841(\mathrm{~F})$ & $\pi(\mathrm{C}-\mathrm{H})$ \\
$829(\mathrm{mF})$ & - & $\pi(\mathrm{C}-\mathrm{H})$ \\
$565(\mathrm{mF})$ & $567(\mathrm{mF})$ & $v(\mathrm{Fe}-\mathrm{C})+\delta(\mathrm{Fe}-\mathrm{C} \equiv \mathrm{O})$ \\
$542(\mathrm{~F})$ & $532(\mathrm{mF})$ & $v(\mathrm{Fe}-\mathrm{C})+\delta(\mathrm{Fe}-\mathrm{C} \equiv \mathrm{O})$ \\
- & $438(\mathrm{f})$ & $v(\mathrm{Fe}-\mathrm{C})$ \\
\hline
\end{tabular}

Intensidades das bandas: $\mathrm{f}=$ fraco $; \mathrm{F}=$ forte $\mathrm{mF}=$ muito forte
A molécula $\mathrm{CO}$ é um bom ligante $\pi$-aceitador, estando ligada ao metal de transição através do átomo de carbono. Geralmente, a configuração mais estável dos complexos organometálicos corresponde a um número total de 18 elétrons de valência $\left(d^{10} s^{2} p^{6}\right)$. Esta condição é denominada "regra dos 18 elétrons". Para um complexo de metal de transição $\mathrm{M}$ de fórmula geral $\left[\mathrm{MX} \mathrm{L}_{\mathrm{b}}\right]^{\mathrm{c}+}, \mathrm{X}=$ ligante monovalente e $\mathrm{L}=$ ligante neutro, o número total de elétrons é expresso pela Equação 1 (método covalente):

Número de elétrons $=\mathrm{N}+\mathrm{a}+2 \mathrm{~b}-\mathrm{c}$

onde, $\mathrm{N}$ corresponde ao grupo do metal.

O cálculo do número de elétrons dos complexos sintetizados neste experimento é apresentado pela Tabela 2. Observa-se que em ambos os casos os complexos seguem a regra dos 18 elétrons, o que indica configuração de máxima estabilidade.

Tabela 2. Cálculo do número total de elétrons de valência pelo método covalente

\begin{tabular}{lcc}
\hline & $\begin{array}{c}{\left[\mathbf{F e}\left(\eta^{5}-\mathbf{C}_{5} \mathbf{H}_{5}\right)\right.} \\
\left.(\mathbf{C O})_{2}\right]_{2}\end{array}$ & $\begin{array}{c}{\left[\mathbf{F e}(\mathbf{C O})_{2}\left[\left(\eta^{5}-\right.\right.\right.} \\
\left.\left.\mathbf{C}_{5} \mathbf{H}_{5}\right) \mathbf{C l}\right]\end{array}$ \\
\hline $\begin{array}{l}\text { Fe, elétrons } \\
\text { de valência }\end{array}$ & 8 & 6 \\
ciclopentadienila & 5 & 6 \\
elétrons CO terminal & 2 & 4 \\
2 CO ponte & 2 & - \\
ligação interatômica & 1 & - \\
ferro-ferro & & 2 \\
ligação com cloro & - & \\
\hline
\end{tabular}

A ligação M-CO envolve um efeito eletrônico sinérgico, onde ao mesmo tempo em que ocorre uma doação $\sigma$ do CO para o M, há também uma retrodoação $\mathrm{M}-\mathrm{CO}$, o que resulta em ligações $\mathrm{M}-\mathrm{CO}$ mais fortes e $\mathrm{CO}$ mais fracas do que se esperariam, se estas interações fossem verificadas isoladamente ${ }^{15}$ (Figura 3). A freqüência vibracional do CO livre ${ }^{12}$, não coordenado, é observada em valor alto, de aproximadamente $2143 \mathrm{~cm}^{-1}$.

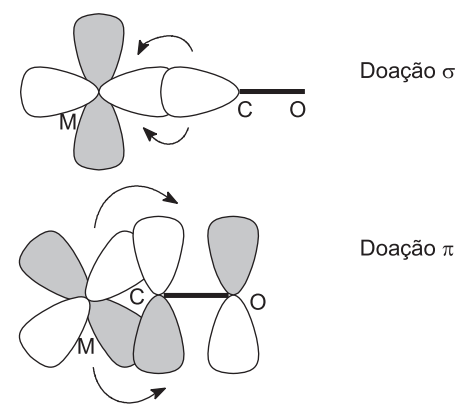

Figura 3. Esquema dos orbitais moleculares envolvidos no efeito sinérgico de transferência eletrônica entre um metal de transição $M$ e o ligante CO. Adaptado da ref. 15

Os estiramentos vibracionais das duas ligações CO terminais para o complexo $\left[\mathrm{Fe}(\mathrm{CO})_{2}\left(\eta^{5}-\mathrm{C}_{5} \mathrm{H}_{5}\right) \mathrm{Cl}\right]$ foram observados em números de onda maiores $\left(1992\right.$ e $\left.2050 \mathrm{~cm}^{-1}\right)$ que em $\left[\mathrm{Fe}(\mathrm{CO})_{2}\left(\eta^{5}\right.\right.$ $\left.\left.\mathrm{C}_{5} \mathrm{H}_{5}\right)\right]_{2}\left(1935 \mathrm{~cm}^{-1}\right)$, conforme pode ser observado na Tabela 1 . Isto se deve à ocorrência de um decréscimo da densidade eletrônica no metal, quando se encontra em um estado formal de oxidação maior, que, por conseqüência, diminui a retrodoação $\mathrm{M}-\mathrm{CO}$, fortalecendo as ligações $\mathrm{CO}$ e enfraquecendo a ligação M-CO. 


\section{CONCLUSÕES}

Uma rota sintética para a obtenção do composto $\left[\mathrm{Fe}(\mathrm{CO})_{2}\left(\eta^{5}-\right.\right.$ $\left.\mathrm{C}_{5} \mathrm{H}_{5}\right) \mathrm{Cl}$ ] validada em aula prática para 30 alunos foi descrita, apresentando como vantagens, um tempo de aplicação menor e alta simplicidade no procedimento, se comparada aos demais métodos descritos na literatura. Além disso, torna-se importante ressaltar o baixo custo dos reagentes empregados, cerca de US\$ 3.00 por aluno, tornando-a ainda mais interessante quanto à sua viabilidade de aplicação em cursos de graduação em Química. Além dos mecanismos das reações e dados espectroscópicos, os complexos de metal de transição também puderam ser abordados em termos estruturais e de ligação química, cujos conceitos reforçam importantes princípios da química de organometálicos, tais como doação-retrodoação eletrônica entre metal-CO e a aplicação da regra dos 18 elétrons de valência.

\section{AGRADECIMENTOS}

Aos alunos da disciplina QG 650 (Laboratório de Síntese Orgânica e Inorgânica) do $2^{\circ}$ semestre de 2004 que realizaram este experimento pela primeira vez com dedicação. Os autores também agradecem ao técnico I. Trigao do laboratório de ensino do Instituto de Química - UNICAMP. À CAPES, ao CNPq e à FAPESP.

\section{REFERÊNCIAS}

1. Davis, S. G.; Walker, J. C.; J. Chem. Soc. Chem. Commun. 1986, 495.

2. Rosan, A.; Rosenblum, M.; J. Org. Chem. 1975, 40, 3621.

3. Rosenblum, M; Scheck, D.; Organometallics 1982, 1, 397.

4. Liu, L.-K.; Eke, U. B.; Mesubi, M. A.; Organometallics 1995, 14, 3958.

5. Liu, L.-K.; Chang, K.-Y.; Wen, Y.-S.; J. Chem. Soc. Dalton Trans. 1998, 741.

6. Piper, T. S.; Cotton, F. A.; Wilkinson, G.; J. Inorg. Nucl. Chem. 1955, 1, 165.

7. Williams, W. E.; Lalor, F. J.; J. Chem. Soc. Dalton Trans. 1973, 1329.

8. Johnson, E. C.; Meyer, T. J.; Winterton, N.; Inorg. Chem. 1971, 10, 1673.

9. Dombeck, D. D.; Angelici, R. J.; Inorg. Chim. Acta 1973, 7, 345.

10. King, R. B.; Organometallic Syntheses, Academic Press: New York, 1965, vol. 1.

11. Coates, G. E; Green, M. L. H.; Powell, P. Wade, K.; Principles of Organometallic Chemistry, Barnes \& Noble Inc.: New York, 1968.

12. Nakamoto, K.; Infrared and Raman Spectra of Inorganic Compounds, $4^{\text {th }}$ ed., John Willey \& Sons Inc: New York, 1993.

13. Hooker, R. H.; Mahmoud, K. A.; Rest, A. J.; J Chem. Soc. Dalton Trans. 1990, 1231.

14. Gansow, O. A.; Schexnayder, D. A.; Kimura, B. Y.; J. Am. Chem. Soc. 1972, 94, 3406.

15. Huheey, J. E; Keiter, E. A.; Keiter, R. L.; Inorganic Chemistry - Principles Structure and Reactivity, $4^{\text {th }}$ ed., Harper Collins College Publishers: New York, 1993. 